\title{
Portable gamma cameras: the real value of an additional view in the operating theatre
}

\author{
Joan Duch
}

Published online: 25 February 2011

(C) Springer-Verlag 2011

It has been a long time since the preliminary portable gamma cameras (PGCs) were developed [1]. During the last decade some PGCs have become available for clinical practice, and can be used in open surgery or with laparoscopy, providing information that can be combined with that coming from conventional or laparoscopic gamma probes. Some of these PGCs are not designed specifically for radioguided surgery (Dilon 6800 gamma camera, a model optimized to perform breast-specific gamma imaging, or Cardiotom 3D mobile gamma camera, optimized to perform myocardial perfusion scintigraphy), but other models are focused on sentinel node (SN) procedures [2-4].

In this issue of the journal, Vidal-Sicart et al. [5] report on the use of the Sentinella S102 (Oncovision) camera, which is a compact scintillation camera with a $50 \times 50 \times$ $4 \mathrm{~mm}^{3} \mathrm{CsI}$ crystal optically coupled through a light guide to a flat panel type position-sensitive photomultiplier tube. This model is connected to a computer via USB, which processes the output signals and provides energy to the detector system. It has three interchangeable pin-hole collimators with apertures of diameter 1.0, 2.5 and $4 \mathrm{~mm}$, and is mounted in a support to facilitate transport to the operating theatre. This support also incorporates a touchsensitive screen, which allows images to be viewed with ease throughout the surgical process. The intrinsic spatial resolution is approximately $2 \mathrm{~mm}$ and the energy resolution

J. Duch $(\bowtie)$

Department of Nuclear Medicine, Hospital Sant Pau,

Barcelona, Spain

e-mail: jduchr@santpau.cat is $13 \%$ at $140 \mathrm{keV}$ [6]. This PGC is provided with laser devices, which can help centre the image acquisitions, and it can also acquire images from dual isotopes. It is able to use a pointer (i.e. of iodine-125) to help localize hot spots seen on the screen.

The main application of these devices is in SN procedures. SNs are widely used in breast cancer, melanoma and head and neck cancer, but in the last decade, their use has expanded to additional indications, with more sophistication and complexity in the technique, including indications in urology, gynaecology and gastroenterology. The addition of PGCs has improved the SN technique, particularly when intraoperative tracer injection is needed or in laparoscopic SN localization [7-9].

In breast cancer, the main indication for a $\mathrm{SN}$ procedure worldwide, usually no laparoscopic technique and no intraoperative tracer injection is needed. The utility of the PGC in breast cancer remains only when no conventional gamma camera is available, and in particular in cases with extra-axillary drainage. In breast cancer, the use of PGCs in radioguided occult lesion localization combined with a conventional gamma probe has been described [7].

In head and neck cancers the usefulness of the PGC seems more clear, because of the unpredictable lymphatic drainage and the possibility of comparing the preoperative image and the intraoperative image in order to ensure that no $\mathrm{SN}$ is missed. This is made possible by PGCs as they can give an overview of all radioactive hot spots within the surgical field. Prior SPECT images may be mandatory in head and neck cancer, with images from the PGC being used to guide the surgeon and to make sure that the $\mathrm{SN}$ has been completely resected. Vermeeren et al. [8] have recently reported an improvement of $24 \%$ in $\mathrm{SN}$ detection. 
The SN procedure has been introduced into urology, in particular for penile, bladder, prostate, testicular and renal cell cancers. The utility of PGC has been tested in prostate, testicular and renal cell cancer, and seems to offer information that assists particularly in the differentiation of SN from second echelon nodes [9-11]. In gynaecology, PGCs can be applied in cervical and endometrial SN procedures, either with open surgery or with laparoscopy. In such indications, PGCs can offer appropriate assessment of the SN detected during surgery. For example, in gynaecological cancer, we currently use the PGC after the surgeon has resected the nodes using the laparoscopic gamma probe to confirm that all hot spots has been adequately removed. In gastrointestinal malignancies we also use the PGC in colorectal SN procedures, although the visualization of the hot spots in such tumours is often hampered by the intense physiological uptake in the colon, with laparoscopic gamma probe detection being more reliable than PGC.

PGCs can also be useful in other kinds of radioguided surgery. For instance in the detection of parathyroid adenomas [12]. It might be easier to detect ectopic than nonectopic parathyroid adenomas, due to the high background usually present in the thyroid area. Another potential application could be the monitoring of leakage during selective limb perfusion (for example, with melphalan plus TNF $\alpha$ ). In this case PGC could work with high sensitivity as a gamma probe, with specific software provided to continuously monitor the possibility of leakage [13].

If PGCs are to provide diagnostic images, significant improvements are still needed. These devices are typically provided with a pin-hole collimator, with a field of view and performance too limited to offer diagnostic images, for example, of bone. At the current stage of development, a possible application would be in the diagnosis of brain death, where imaging with a PGC can be performed in the critical care unit avoiding the transfer of the patient to the nuclear medicine service [14]. In order to improve the imaging quality, new collimators may be developed soon.

In the study by Vidal-Sicart et al. [5], lymphatic mapping with a PGC was compared to conventional gamma camera imaging in breast cancer preoperative lymphoscintigraphy. The authors found that the PGC was able to depict SNs in $88 \%$ of cases, in combination with a lead shield to mask the injection site, and may be useful in centres without the possibility of acquiring a preoperative image with a conventional gamma camera.

There are several issues to underline in this article. Firstly, in breast cancer SN procedures the PGC is in principle less useful, as lymphoscintigraphy in these patients is more predictable than in other SN procedures. It might be useful to exclude extra-axillary drainage, but
SPECT CT already offers a reliable means to localize the extra-axillary lymph nodes. The authors indicate that PGCs can reduce the workload of conventional gamma cameras, but planar preoperative lymphoscintigraphy is currently done in less than 5 minutes, thus the impact of PGCs on resource utilization would be minimal. Secondly, from the results of this prospective study, the authors suggested that a lead mask should always be used in order to obtain the same sensitivity as conventional gamma cameras. However, it seems that intramammary SNs could then be missed as they would stay outside the effective field of view. Moreover, two or three images might be needed in obese patients, in order to adequately cover the whole region, which is clearly less practical than using a conventional gamma camera. Finally, the authors did not find any SN that was not detected by a conventional gamma camera but was detected by the PGC. Therefore it is difficult to see the added value of using a PGC, unless a conventional gamma camera is not available.

In summary, as has been previously indicated [15], 'better eyes are always welcome in radioguided surgery' to optimize disease management. The increasing indications for the SN procedure combined with the emerging techniques of radioguided surgeries further support the need for improved PGCs.

\section{References}

1. Hurwitz S, Ashburn W, Green J, Halpern S. Clinical applications of a portable scintillation camera. J Nucl Med. 1973;14:585-7.

2. Tanaka C, Fujii H, Shiotani A, Kitagawa Y, Nakamura K, Kubo A. Sentinel node imaging of laryngeal cancer using a portable gamma camera with CdTe semiconductor detectors. Clin Nucl Med. 2005;30:440-3.

3. Scopinaro F, Tofani A, Di Santo G, Di Pietro B, Lombardi A, Lo Russo M. High-resolution hand-held camera for sentinel-node detection. Cancer Biother Radiopharm. 2008;23:43-52.

4. Mathelin C, Salvador S, Huss D, Guyonnet L. Precise localization of sentinel lymph nodes and estimation of their depth using a prototype intraoperative mini gamma-camera in patients with breast cancer. J Nucl Med. 2007;48:623-9.

5. Vidal-Sicart S, Vermeeren L, Solà $\mathrm{O}$, Paredes $\mathrm{P}$, Valdés-Olmos R. The use of a portable gamma camera for preoperative lymphatic mapping: a comparison with a conventional gamma camera. Eur J Nucl Med Mol Imaging. 2011. doi:10.1007/s00259-010-1682-z.

6. Sánchez F, Fernández MM, Giménez M, Benlloch JM, RodríguezAlvarez MJ, García de Quirós F, et al. Performance tests of two portable mini gamma cameras for medical applications. Med Phys. 2006;33:4210-20.

7. Paredes P, Vidal-Sicart S, Zanón G, Roé N, Rubí S, Lafuente S, et al. Radioguided occult lesion localisation in breast cancer using an intraoperative portable gamma camera: first results. Eur J Nucl Med Mol Imaging. 2008;35:230-5.

8. Vermeeren L, Valdés-Olmos R, Klop W, Balm A, Van den Brekel W. A portable gamma-camera for intraoperative detection of sentinel nodes in the head and neck region. J Nucl Med. 2010;51:700-3. 
9. Vermeeren L, Valdés-Olmos R, Meinhardt W, Bex A, Van der Poel $\mathrm{H}$, Vogel $\mathrm{W}$, et al. Intraoperative radioguidance with a portable gamma camera: a novel technique for laparoscopic sentinel node localisation in urological malignancies. Eur J Nucl Med Mol Imaging. 2009;36:1029-36.

10. Vermeeren L, Meinhardt W, Van der Poel H, Valdés Olmos R. Lymphatic drainage from the treated versus untreated prostate: feasibility of sentinel node biopsy in recurrent cancer. Eur J Nucl Med Mol Imaging. 2010;37:2021-6.

11. Bex A, Vermeeren L, de Windt G, Prevoo W, Horenblas S, Valdés Olmos R. Feasibility of sentinel node detection in renal cell carcinoma: a pilot study. Eur J Nucl Med Mol Imaging. 2010;37: 1117-23.
12. Ortega J, Ferrer-Rebolleda J, Cassinella N, Lledo S. Potential role of a new hand-held miniature gamma camera in performing minimally invasive parathyroidectomy. Eur J Nucl Med Mol Imaging. 2007;34:165-9.

13. Orero A, Vidal-Sicart S, Roé N, Muxí A, Rubí S, Duch J, et al. Monitoring system for isolated limb perfusion based on a portable gamma camera. Nuklearmediziner. 2009;48:166-72.

14. Calvo C, De la Riva PA, Cambil T, Alvarez E, Castro J. Brain perfusion image with a portable mini-gamma camera (Sentinella) in brain death. Rev Esp Med Nucl. 2009;28:83-4.

15. Valdés Olmos R, Vidal-Sicart S, Nieweg O. Technological innovation in the sentinel node procedure: towards 3-D intraoperative imaging. Eur J Nucl Med Mol Imaging. 2010;37:1449-51. 